\title{
Tabebuia heterophylla (DC) Britton; données nouvelles sur la reproduction sexuée et végétative
}

\author{
R. HUC, M. BARITEAU \\ INRA, Département des Recherches forestières \\ Station de Recherches forestières, Groupe régional de Recherches de Guyane \\ Avenue de France, BP 709, 97387 Kourou Cedex, Guyane
}

\begin{abstract}
Résumé
Des relevés de terrain ont été effectués afin d'étudier la phénologie d'une espèce des forêts de basse altitude des Antilles : le Tabebuia heterophylla. Parallèlement un stockage à basse température a pu étendre à huit mois la longévité des graines. Une technique simple de bouturage est mise au point.
\end{abstract} Caraïbes.

Mots clés: Tabebuia heterophylla, pouvoir germinatif, phénologie, bouturage, zone tropicale,

\section{Introduction}

La nécessité de reboisement des terres libérées par l'agriculture ou des zones trop sèches dans les Antilles a conduit les Services forestiers à rechercher des essences adaptées à ces conditions particulières. Le "Poirier-Pays" (Tabebuia heterophylla) retient l'attention des aménagistes pour ses facultés à occuper des milieux bioclimatiques très variés dans la zone concernée.

Pour cette espèce, qui a rarement fait l'objet de plantations systématiques, les données sur la biologie et la croissance sont relativement rares (WADSWORTH, 1945). Les aspects écophysiologiques ont commencé récemment à être abordés (BorcherT, 1983 ; Huc, 1985) et font état des aptitudes de l'arbre pour l'évitement de la sécheresse. Nous nous sommes intéressés ici à apporter des données utiles pour le reboisement et concernant la phénologie, la conservation des graines et la multiplication végétative.

\section{Matériel et méthodes}

Pour les études de phénologie, trois peuplements ont été retenus afin d'y effectuer des observations régulières et bimensuelles sur la reproduction sexuée, sur les caractères végétatifs apparents, et sur la croissance en diamètre par rubans dendrométriques. Les 
localités correspondent à la région peu arrosée du Nord du plateau de Grande-Terre (Sans-Fenêtre, Anse-Bertrand) ; à celle de la côte sous-le-vent "sèche » de la BasseTerre (Morphy, Pointe-Noire) ; et à celle de la coite-au-vent très arrosée (Sainte-Marie, Capesterre-Belle-Eau).

Les essais sur la conservation des graines ont porté d'une part sur la comparaison entre provenances, à température ambiante et à $15^{\circ} \mathrm{C}$ et d'autre part sur les graines issues d'un même arbre et conservées, sous sacs scellés:

- à température ambiante avec ou sans insecticide (Malathion) ;

- à $5{ }^{\circ} \mathrm{C}$ avec ou sans Malathion + Silicagel ;

- à $2,5^{\circ} \mathrm{C}$ avec Malathion + Silicagel.

Dans la comparaison entre provenances. 50 graines sont mises à germer toutes les semaines pour chaque variété. Dans les essais survants 30 graines sont mises à germer tous les 60 jours.

Les bouturages sont effectués dans du sable de rivière à partir des articles terminaux des branches, sur des sections à deux nœuds. La moitié du limbe des feuilles est conservée. Les traitements sont pratiqués avec des concentrations d'A.I.B. variées ou de la Rootone. La serre de bouturage est un tunnel plastique équipé d'un système rudimentaire de brumisation (un cycle de $45 \mathrm{sec}$. environ tous les $1 / 4$ d'heure).

\section{Résultats}

\subsection{Phénologie (cf. tabl. 1)}

\subsection{Chute des feuilles}

L'importance du phénomène est variable selon la zone bioclimatique. Ainsi en zone bien arrosée, la défoliation est partielle et alle dure d'octobre à février (c'est le cas à Sainte-Marie). En zone de saison sèche bien marquée la chute des feuilles est totale et associée à celle-ci.

\subsection{Floraison et fructification}

Comme pour la défoliation l'intensité de la floraison est variable selon les régions. Ainsi en côte au vent la floraison reste assez discrète avec des pics plus ou moins marqués dont un très net en début de saison des pluies. En zone plus sèche, la floraison est spectaculaire et coïncide avec l'arrêt de la sécheresse. Les fruits arrivent à maturité sept semaines après l'apparition des boutons floraux.

\subsection{Croissance en diamètre}

Au cours d'un cycle annuel, les mesures faites au microdendromètre montrent que laugmentation en diamètre du tronc est étroitement lié à la recrudescence des précipitations. Un retrait s'opère en saison sèche. Il est d'autant plus accusé que cette saison est longue et peu arrosée. 
TABLIEAU 1

Caractères phénologiques du Tabebuia heterophylla dans les trois stations étudiées.

Phenological characteristics of Tabebuia heterophylla in the three studied sites.

\begin{tabular}{|c|c|c|}
\hline & & Aou. Sep. Oct. Nov. Dec. Jan. Fév. Mar. Avr. Mai Jun. Jul. \\
\hline $\begin{array}{l}\text { Zone } 1 \text { (sèche) } \\
\text { Sans fenêtre } \\
\text { Nord Grandc-Terre }\end{array}$ & $\begin{array}{l}\mathrm{fl} \\
\mathrm{fr} \\
\mathrm{ch} \\
\mathrm{nf} \\
\mathrm{rd}\end{array}$ & * Saison des pluies __ $\_$saison sèche $\stackrel{\text { transition } \longrightarrow}{\longleftrightarrow} \longleftrightarrow$ \\
\hline $\begin{array}{l}\text { Zone } 2 \text { (sèche) } \\
\text { Morphy } \\
\text { Cóte s/le vent }\end{array}$ & $\begin{array}{l}\text { fl } \\
\text { fr } \\
\text { ch } \\
\text { nf } \\
\text { rd }\end{array}$ & 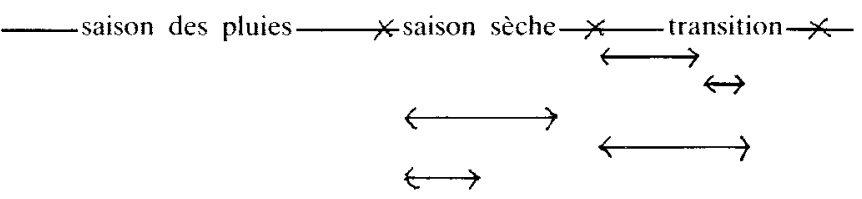 \\
\hline $\begin{array}{l}\text { Zone } 3 \text { (humide) } \\
\text { Sainte-Marie } \\
\text { Cote-au-vent }\end{array}$ & $\begin{array}{l}\mathrm{fl} \\
\mathrm{fr} \\
\mathrm{ch} \\
\mathrm{nf} \\
\mathrm{rd}\end{array}$ & 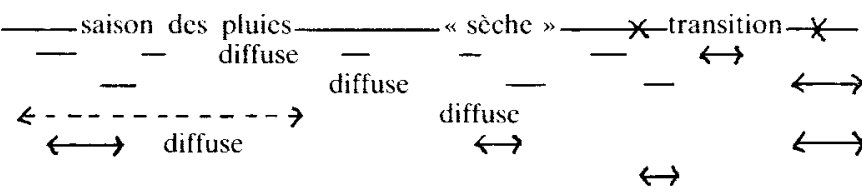 \\
\hline
\end{tabular}

$\mathrm{fl}=$ floraison $; \mathrm{fr}=$ fructification $; \mathrm{ch}=$ chute des feuilles $: \mathrm{nf}=$ formation de nouvelles feuilles ; rd = arrêt de croissance en diamètre: retrait.

La croissance moyenne annuelle est voisine de $1 \mathrm{~cm}$ par an pour les plantations depuis l'origine. A Morphy, la croissance relevée du 25/01/83 au 8/01/85 a été de $1,9 \mathrm{~cm}$.

\subsection{Conservation des graines}

a) A température ambiante et quelle que soit la provenance, la longévité de la graine n'excède pas 80 jours.

b) Stockées à $15^{\circ} \mathrm{C}$, les graines maintiennent plus longtemps leur pouvoir de germination : 60 p. 100,80 p. 100 et 90 p. 100 respectivement pour les provenances à 1,3 et 5 folioles au bout de 90 jours.

c) A $5{ }^{\circ} \mathrm{C}$, sans insecticide ni dessicateur, le pouvoir germinatif est préservé totalement jusqu'à 240 jours. Cette température (qui peut être celle du compartiment à légumes d'un réfrigérateur) est recommandée pour la conservation.

\subsection{Multiplication végétative}

Les essais de bouturage montrent que la qualité des enracinements est meilleure pour des doses d'A.I.B. de 0,5 ou 1 p. 100 (près de 100 p. 100 d'enracinement). Après repiquage, la croissance des boutures est très bonne. 
Cette technique rudimentaire de bouturage qui donne sur le "Poirier-Pays » de très bons résultats pourra être utilisée directement dans le programme d'amélioration génétique de l'espèce. La sauvegarde des beaux individus menacés peut ainsi être assurée après rajeunissement (greffages).

\title{
Conclusions
}

Il apparaît que l'obtention de matériel pour le reboisement ne rencontre pas d'obstacle majeur. Nous avons pu montrer que la durée de vie des semences du "Poirier-Pays" pouvait être prolongée par une méthode peu coûteuse puisque un simple stockage au réfrigérateur suffit pour l'étendre de 2 à 8 mois ou davantage. La prévision des périodes de fructification doit permettre de faciliter la planification des récoltes. En outre la reproduction de l'espèce par bouturage apparaît relativement aisée.

Si l'orientation prise pour le reboisement se confirme, le problème majeur restera celui de l'obtention de beaux sujets. La sélection à rebours qui s'est opérée sur cette essence par suite d'une exploitation abusive a rendu très rares les individus bien conformés (troncs rectilignes et non canelés, absence de fourches basses...). Toutefois le «Poirier-Pays» présente d'autres caractéristiques que nous avons étudiées dans un travail qui sera publié ultérieurement, telles que : une forte variabilité naturelle, une reproduction sexuée précoce et abondante. La mise en route d'un programme d'amélioration est facilitée par ces aptitudes particulières.

L'évolution tragique de certaines îles voisines touchées par un déboisement excessif permet de situer parfaitement, s'il en était besoin, l'enjeu d'une telle recherche.

\author{
Reçu le 18 février 1987. \\ Accepté le 18 février 1987.
}

\section{Remerciements}

Les auteurs remercient particulièrement $M$. Patrick LABBE représentant la station de Recherches forestières de I'INRA en Guadeloupe ainsi que les responsables et le personnel technicue de rOffice National des Forêts de Guadcloupe qui ont permis la localisation des sites d'étude et la réalisation technique du travail. 


\section{Summary}

Tabebuia heterophylla (DC) Britton : new data on breeding and vegetative propagation

Field researches have been conducted on the phenology of a tree species of the West Indies lowland forests : Tabebuia heterophylla. Conjunctly, by a conservation at low temperature, the seed viability has been extended from 2 up to 8 months. A method of propagation by cuttings, easy to use, is proposed here.

Key-words: Tabebuia heterophylla, longevity of seeds, phenology, propagation by cuttings, tropical zone, West Indies.

\section{Références bibliographiques}

Borchert R., 1983. Phenology and control of flowering in tropical trees. Biotropica, 15 (2), 81-89.

Huc R., 1985. Premiers résultats expérimentaux sur le comportement de Tabebuia heterophylla (D.C.) Britton et de Hymenaea courbaril L. vis-à-vis de la sécheresse. Ann. Sci. For., 42 (2), 201-224.

WADSWORTH F.H., 1945. Further notes on the regeneration and growth of Tabebuia pallida Miers. Caribb. For., 6 (4), 267-269. 